with hypocellular areas and nuclear atypia-s/o ancient schwannoma.

Discussion JF mass can be classified according to the structure of origin; it was to be extrinsic or intrinsic. Based on history and imaging characteristics, the diagnosis was thought to be GJ. But histopathological diagnosis was AS. There are four variants of schwannoma. AS is characterized by degenerative changes, such as hemorrhage, cystic degeneration, hyalinization and calcification. These characteristics mimic GJ on imaging giving salt and pepper appearance. Treatment is surgical excision since it is a benign tumor and radioresistant with good prognosis.

Conclusion Schwannomas of JF though rare has to be considered as a differential diagnosis for JF mass; however, the treatment remains the same with good prognosis.

\section{A0046: Facial Nerve Decompression}

Sweta Mary

In case of trauma or accidents, patients can have temporal bone fracture resulting in facial nerve palsy. Temporal bone fractures, blunt or penetrating head and neck trauma, and iatrogenic surgical injury are all common causes for facial nerve injury. Immediate paralysis is suggestive of nerve disruption or severe compression, such as bone fragment. Temporal bone fractures are classified as longitudinal or transverse or mixed. It is described as nerve swelling within the bony canal. The odema and inability to expand beyond the bony confines creates a conduction block which prevents the axoplasmic flow. In case of temporal bone fracture, the treatment for facial nerve palsy depends on the fracture location and the ideal time for surgery following trauma. In our OPD, a patient came with alleged history of trauma following which he had facial nerve palsy. We performed facial nerve decompression and patient had a good prognosis, postoperatively. Facial nerve decompression is a surgery recommended for complete facial nerve palsy in which patients get benefitted by regaining the facial nerve functions. Surgical decompression of all nerve segments affected by temporal bone fracture should be performed using middle cranial fossa approach or transmastoidal approach or translabyrinthine approach.

\section{A0047: Association of Central Obesity with the Severity and Audiometric Configurations of Age-Related Hearing Impairment \\ Utkarsh Agrawal, ${ }^{1}$ R.K. Jain ${ }^{1}$ \\ 'IMS, BHU, Varanasi, Uttar Pradesh, India}

Aim 1. To know the relationship between central obesity with age-related hearing loss.

2. To know the most important indicator of central obesity associated with age-related hearing loss.

3. To know the relationship if any between age, degree of obesity, and configuration of hearing loss.

4. To know the relationship between diabetes mellitus, hypertension with the central obesity, and severity of hearing loss.

Material and Methods This is a prospective, randomized, single blind study performed over a period of
2 years from August 2016 to July 2018. Six hundred patients who attended outpatient department (OPD) in the Department of Otorhinolaryngology, with sensorineural hearing loss following inclusion criteria, are included in the study. Detailed history was taken followed by clinical examination. All patients were subjected to complete ENT examination, pure tone audiometric examination was done in all cases (MAICO MA42 audiometer). Height, body weight, and waist circumference (WC) were measured.

Results The correlation between obesity and SNHL was found to be more significant in females than males. Obese and diabetic patients of both sexes were found to have higher degree of hearing loss for both high and low frequencies as compared with nonobese patients. Good correlation was found between BMI and degree of hearing loss. Abrupt high tone loss type of audiometric configuration was the commonest finding in both male and females.

Conclusion There is a correlation between ages related to sensory neural hearing loss with central obesity and its comorbidities, with waist circumference as the most important indicator.

Clinical Significance A careful understanding of the modifiable risk factors for age-related sensory neural hearing loss help in its early detection and prevention.

\section{A0048: The Depth of Facial Nerve in the Mastoid Bone Praveen Kumar B. Y., ${ }^{1}$ Vanlal Hriati Leivang ${ }^{1}$ \\ ${ }^{1}$ Department of ENT, Mysore Medical College and Research Institute, Mysuru, Karnataka, India}

Background The hallmark of the temporal bone is variation. Various important structures like the facial nerve run in the temporal bone at various depths can be injured during mastiodectomy.

Method Twenty wet cadaveric temporal bone were dissected. A cortical mastoidectomy was performed followed by a canal wall down mastiodectomy and the depth of the vertical segment of the facial nerve in the mastoid was determined.

Results The mean depth of the second genu was $13.8 \mathrm{~mm}$. The mean depth of the stylomastiod foramen was $12.75 \mathrm{~mm}$ and the mean distance from the annulus at 6'0 clock to the stylomastoid foramen was $10.22 \mathrm{~mm}$.

Conclusion There is significant variation in the average depth of the facial nerve in the mastoid.

\section{A0049: Comparative Study between Microscopic Myringoplasty and Endoscopic Myringoplasty}

Aim To compare the outcomes of microscope-assisted postauricular approach tympanoplasty using temporalis fascia and endoscope-assisted endomeatal tympanoplasty using tragal perichondrium, among patients with CSOM.

Materials and Methods In 81 patients, group A consisted of 40 patients who underwent endoscopic myringoplasty and group B consisted of 41 patients who underwent the conventional microscopic technique, the outcome was analyzed in terms of the hearing gain, duration of surgery, graft success rate, and to study the comparative benefits of endoscopic tympanoplasty over microscopic tympanoplasty 